\title{
MEMS MICROSHUTTER ARRAY SYSTEM FOR JAMES WEBB SPACE TELESCOPE
}

M.J. Li, T. Adachi, S.R. Babu, S. Bajikar, M.A. Beamesderfer, R. Bradley, N.P. Costen, K. Denis, A.J. Ewin, R. Fettig, D. Franz, L. Hess, R. Hu, K. Jackson, C.A. Jhabvala, M.D. Jhabvala, D. Kelly, T.T. King, G. Kletetschka, A.S. Kutyrev, B.A. Lynch, T. Miller, S.H. Moseley, V. Mikula, B. Mott, L. Oh, J. Pontius, D. Rapchun, C. Ray, K. Ray, E. Schulte, D.S. Schwinger, P.K. Shu, R.F. Silverberg, W.W. Smith, S. Snodgrass, D. Sohl, L.M. Sparr, R. Steptoe-Jackson, V. Veronica, L.L. Wang, Y. Zheng, C. Zincke NASA Goddard Space Flight Center, Greenbelt MD 20771 USA

\section{ABSTRACT}

A complex MEMS microshutter array system has been developed at NASA Goddard Space Flight Center (GSFC) for use as a multi-object aperture array for a Near-Infrared Spectrometer (NIRSpec), one of the four major instruments carried by the James Webb Space Telescope (JWST). The microshutter arrays (MSAs) are designed for the selective transmission of light (0.6 to $5 \mu \mathrm{m}$ ) with high efficiency (100 times over current technology) and high contrast $(>2000)$. The MSAs are close-packed shutters (100x200 $\mu \mathrm{m}$ pixel size), hybridized with ASIC584 driving components, and packaged using a novel single-side indium bump bonding technology. The MSA system, a mosaic of 2x2 MSA quadrants, is operated with magnetic actuation and electrostatic latching and addressing. Complete MSA quadrant assemblies have been successfully manufactured and fully functionally tested.

\section{INTRODUCTION}

A complex MEMS microshutter array system has been developed at NASA GSFC for use as a multi-object aperture array for a NIRSpec. The NIRSpec is one of the four major instruments carried by JWST, the next generation of space telescope after the Hubble Space Telescope retires. The MSAs are designed for the selective transmission of light with high efficiency and high contrast. It is demonstrated in Figure 1 how a MSA is used as a

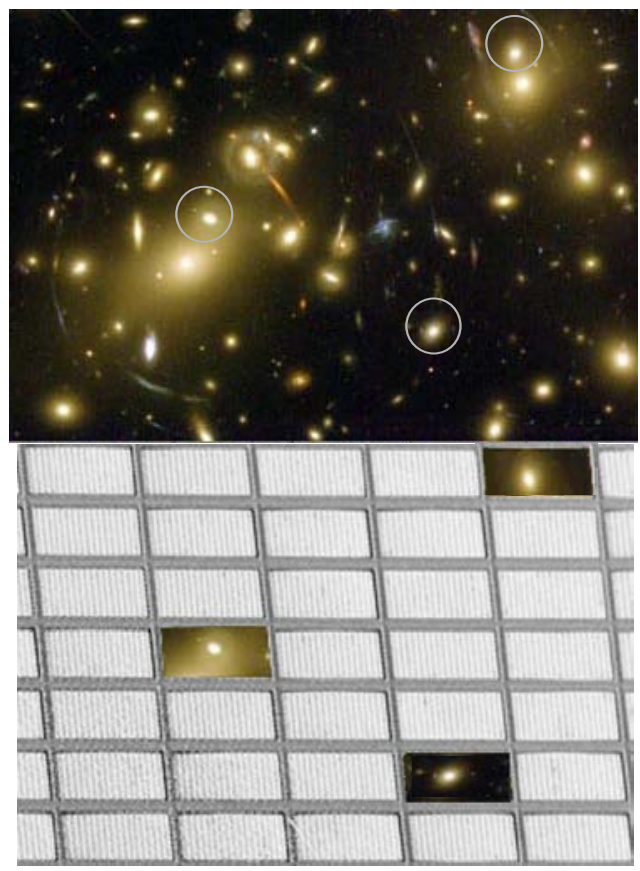

Figure 1. Demonstration of a microshutter array used as an aperture (bottom) to select multiple objects from sky (top) multiple object selector in deep space. The MSAs empower the NIRSpec instrument simultaneously collect spectra from more than 100 targets therefore increases the instrument efficiency 100 times or more. The MSA technology is one of three major innovations on JWST and the first major MEMS devices serving observation missions in space. The NIRSpec instrument is being built-up by a collaborative effort among NASA, the European Space Agency (ESA), and the Canadian Space Agency (CSA). The JWST launching is scheduled for 2013.

\section{MICROSHUTTER ARRAY DESIGN}

Major scientific requirements ${ }^{1}$ for the MSA system include: random access addressing, -- must allow the opening of any shutter distribution and equal to or more than 100 objects simultaneously targeted; field of view, -- must cover the field of view of NIRSpec (3.4' $x$ 3.4'); contrast, --must have an open to closed transmission ratio of $>2000$; life time, -- must operate for $9.4 \times 10^{4}$ cycles with minimal failures; and operating environment, -- must operate in the JWST test and operation environments, including the entire $300 \mathrm{~K}$ to $35 \mathrm{~K}$ temperature range, the power dissipation of $40 \mathrm{~mW}$ average (at $35 \mathrm{~K}$ ), a volume fitting the envelope in NIRSpec instrument, a mass of 10 $\mathrm{kg}$, and the radiation within a life dose of $48 \mathrm{kRad}$. To meet the requirements, the MSA flight concept consists of a MEMS mosaic of $2 \times 2$ format of four fully addressable $365 \times 171$ arrays fitting in the JWST optical path at the focal plane. The MSA system has

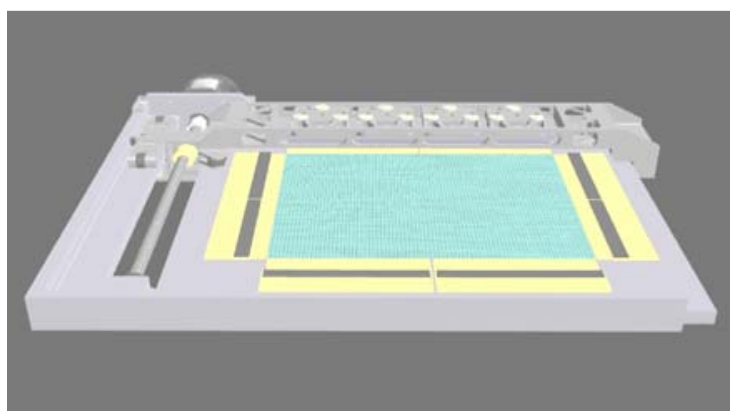

Figure 2. Schematic of an early model for demonstration of the MSA actuation mechanism. A linear magnet sweeps in y direction across the array

a mechanism of magnetic actuation and electrostatic latching and addressing ${ }^{1,2}$. Conductive thin films are tailored on the top of shutters as column electrodes and on the sidewalls in shutter frame windows as row electrodes, respectively. A linear permanent magnet aligned with the shutter rows is positioned on the top of a flipped up-side-down array, as demonstrated in Figure 2 , and sweeps across the array in a direction parallel to shutter rows (moving towards readers). As the magnet sweeps across the 
array, sequential rows of shutters are rotated from their natural horizontal orientation to a vertical open position, where they can be latched to the row electrodes on the sidewalls by a DC bias, typically $40 \mathrm{~V}$. As the bias is removed or insufficient, shutters return to their horizontal closed position upon an elastic restoring force from a torsion flexure on the shutters (see next section).

\section{MICROSHUTTER ARRAY FABRICATION}

As shown in Figure 3, each MSA is fabricated out of a 4" silicon-on-insulator (SOI) wafer using MEMS bulkmicromachining technology. Individual shutters are close-packed
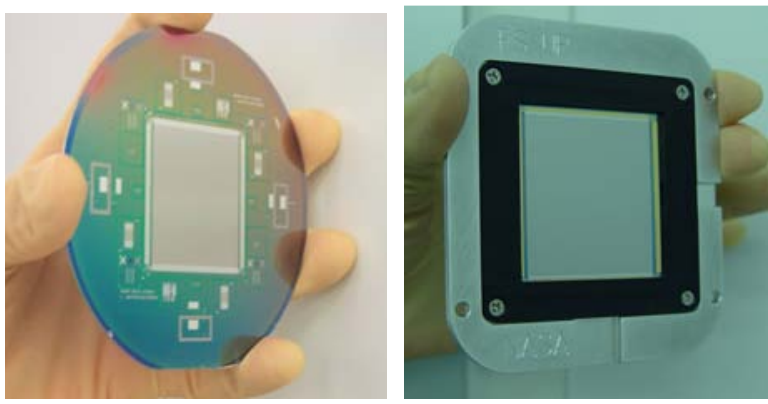

Figure 3. A flight-format $365 \times 171$ microshutter array (right) is fabricated out of a 4" SOI wafer (left)

silicon nitride membranes with a pixel size close to 100x200 $\mu \mathrm{m}$ (Figure 4). Shutters are patterned with a fine torsion flexure that connects to the frame permitting shutters to open 90 degrees with a minimized mechanical stress concentration. Silicon nitride is utilized as the shutter blades based on its excellent mechanical

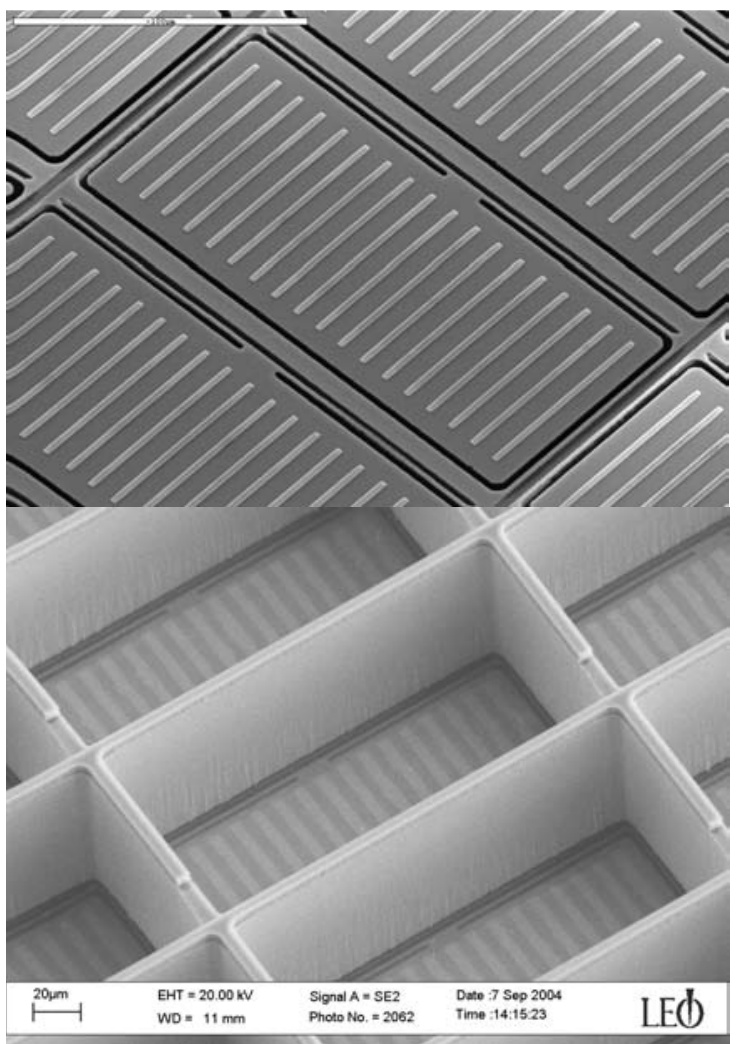

Figure 4: SEM images of the front side (top) and backside (bottom) of a $64 x 128$ microshutter array with zoom-in images of shutter cells. and thermal mechanical performances ${ }^{2}$. A cobalt-iron thin film processed on shutter blades functions as the magnetic element that responds to the external magnetic field for actuating shutters. The thin film is patterned into stripes so to make the magnetic domains in the film parallel to the external magnetic field as much as possible. The design helps to minimize uneven motion of shutters during actuations. In order to prevent light leak, light shields are fabricated on to the surrounding frame of each shutter to cover the gaps between the shutters and the frame, as shown in Figure 5(a). Sub-micron bumps and micro-ribs are tailored on light shields and back walls, respectively, to prevent sticktion, as shown in Figures 5(a) and (b).

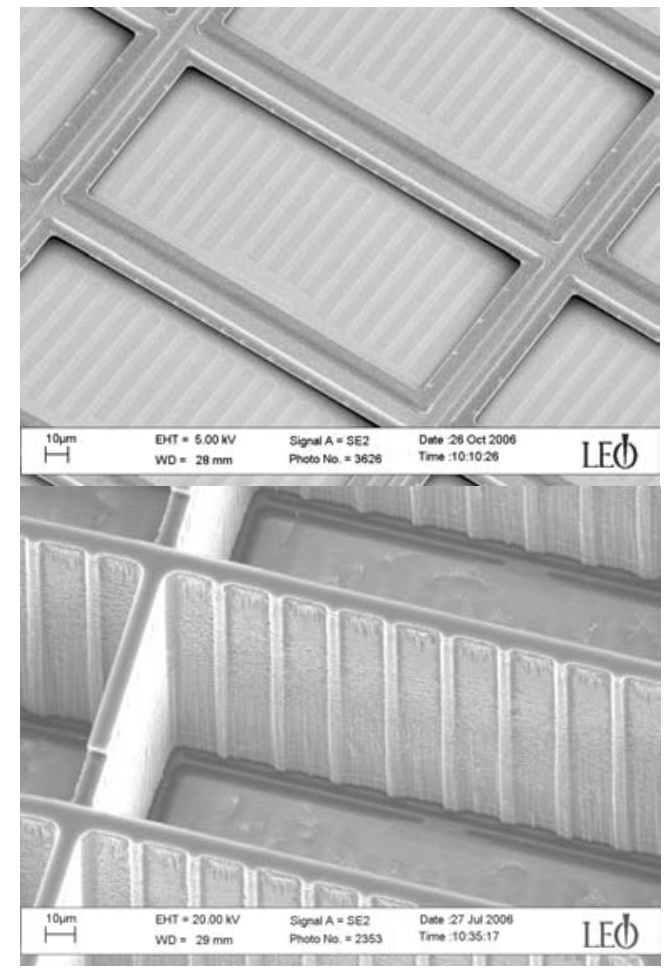

Figure 5. Light shields are patterned to cover the gaps between shutters and frame (top); sub-micron-bumps are made on light shields (top), and micron-ribs on back walls to prevent sticktion (bottom).

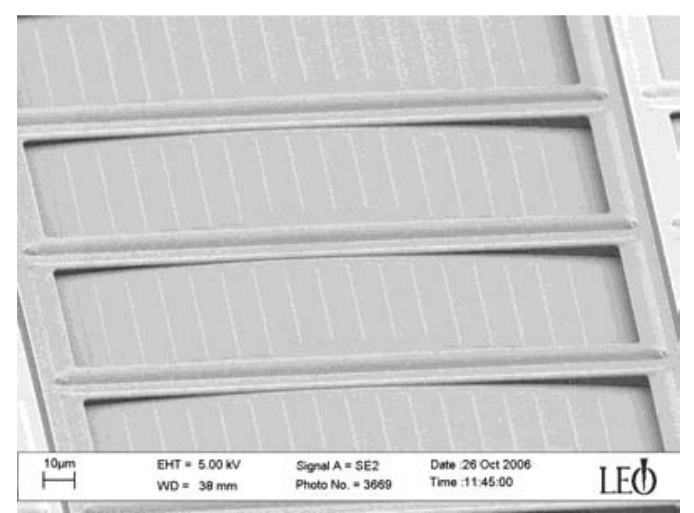

Figure 6. Shutters are slightly bowing down at room temperature and will become flat when the temperature reaches $35 \mathrm{~K}$

JWST instruments are required to operate at cryogenic temperatures as low as $35 \mathrm{~K}$, though they are to be subjected to 
various levels of ground tests at room temperature. The shutters should therefore maintain nearly flat in the entire temperature range between $35 \mathrm{~K}$ and $300 \mathrm{~K}$. An optically opaque and electrically conductive metal-nitride thin film is utilized as a coating material deposited on the shutters with the best thermalexpansion matching to silicon nitride -- the shutter blades themselves. A shutter image shown in Figure 6 was taken at room temperature, presenting shutters slightly bowing down as expected. Shutters become flat when the temperature decreases to $35 \mathrm{~K}$.

\section{PACKAGING}

MSA substrates are made of single crystal silicon, in the thickness of $2 \mathrm{~mm}$, for the best thermal match to the shutter arrays. The bonding between a MSA and a substrate is conducted using a novel single-sided indium flip-chip bonding technology ${ }^{3}$. The indium pumps function as mechanical support as well as electrical paths that connect the column electrodes on a MSA to its substrate. Indium bumps, as shown in Figure 6, are patterned on a substrate through a lift-off patterning process. There are 180,000 indium bumps for attaching a flight-format MSA array to its substrate. Single-side indium bump patterning on the substrate eliminates the risk of damaging MSAs in the lift-off process. Further advantages are no heating or under-fill required in the bonding process that minimize the thermal stress and simplify the packaging process. This bonding technology permits the flatness over the entire MSA within $1 \mu \mathrm{m}$.

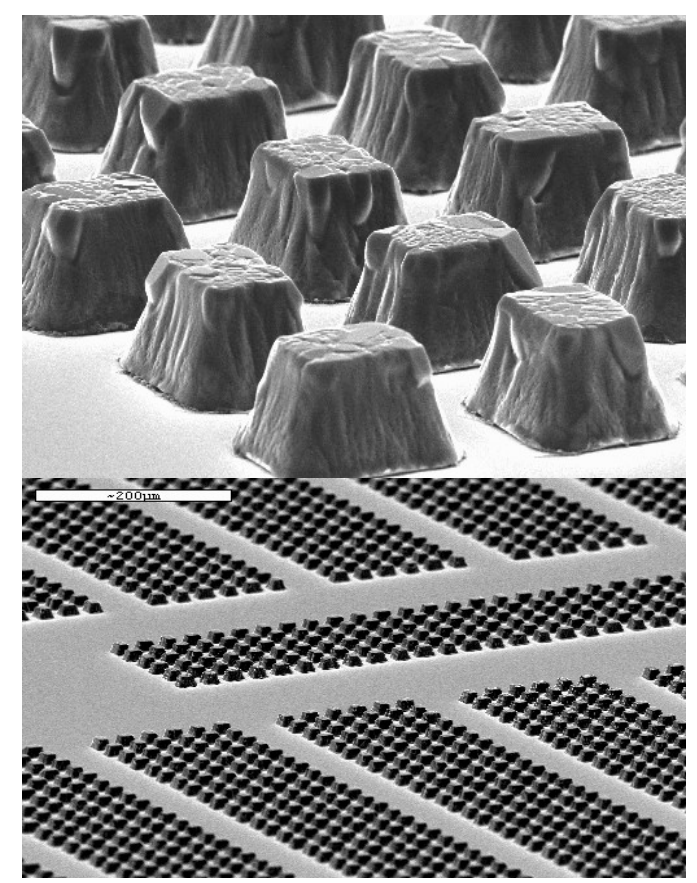

Figure 6. Indium bumps (top) are patterned on a MSA substrate (bottom) for flip-chip bonding between MSAs and their substrates

One of the four quadrants in a $2 \times 2$ mosaic MSA system is shown in Figure 7. The substrate is fully populated with 2-dimensional addressing components. Besides a MSA, each substrate houses five customer-designed ASIC (Application Specific Integrated Circuit) multiplexer/address chips, twenty capacitors, two temperature sensors, numbers of resistors and all necessary inter-

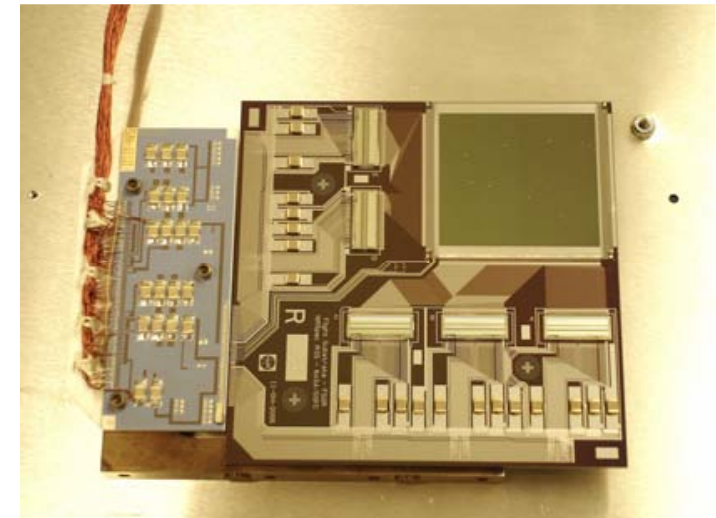

Figure 7. A MSA quadrant assembly consists of a MSA array (top right), a silicon substrate populated with ASIC 2-D addressing components, a daughter board (left), and a flexure (underneath the substrate)

connects. The substrate is electronically connected to a daughter board. Both the substrate and the daughter board are mounted on a flexure that can be seen underneath the substrate. Flexures are designed with the consideration of protecting the silicon substrates from thermal stresses introduced by CTE mismatch between the substrates and a large metal plate. The metal plate is the foundation of the MSA system, housing all four quadrants. A permanent praseodymium quadrupole magnet is designed for the actuation of all four $171 \times 365$ MSA arrays.

\section{QUALIFICATION TESTING}

A series of qualification inspection and testing are conducted at the MSA level and the quadrant level, respectively. The qualification inspection includes the prescreening, open/close test, electrical test, and bowing test, while the qualification testing covers the 2-D addressing, life cycling, optical test, and environmental tests.

In the MSA open/close testing, two failure modes are characterized: the failed open and the failed close. The former is defined as the light leak through a supposedly closed shutter, while the latter as the light blocked by a supposedly open shutter.
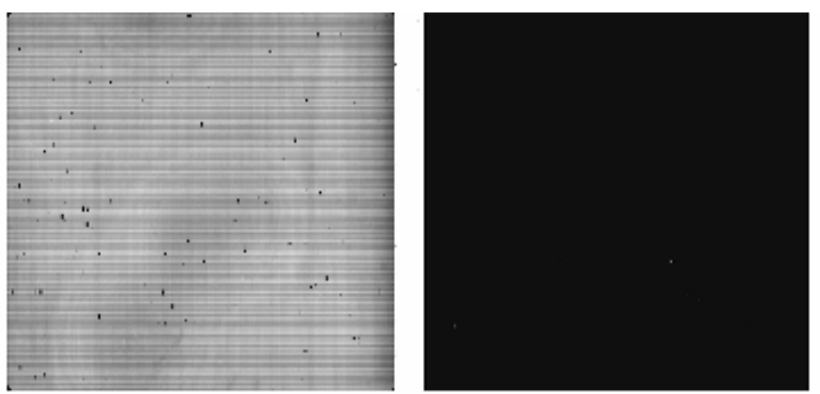

Figure 8. Images of a MSA in an open/close test. A homogenous magnetic force is applied keeping shutters open (left), and withdrawn so to release shutters to their close position (right). Shutters in failed-open/failed-close are enumerated.

In the NIRSpec instrument is required typically the failed-closed shutters $<5 \%$ and the rows with failed-open shutters $<3 \%$ at the beginning of the life (BOL), and the failed-closed shutters $<20 \%$ and rows with failed-open shutters $<6 \%$ at the end of the life (EOL). Before a life cycling test, MSAs are exposed to a 
homogenous magnetic field. Failed-closed and failed-open shutters are counted and recorded automatically. Over thousands of photos are taken from each MSA with details of individual shutters for the records at BOL that will be compared with those taken at EOL. Two images, as shown in Figure 8, present the failed-closed and failed-open shutters in a flight-format MSA (171x365 pixels) with 150 failed-closed shutters $(0.2 \%)$ and 3 failed-open shutters (1.8\%). Shutter bowing tests are conducted using a confocal imaging system. Shutter bowing is monitored over a temperature span typically from $300 \mathrm{~K}$ to $35 \mathrm{~K}$ with a resolution of $0.1 \mathrm{um}$. Bowings along the short and long dimensions of shutters, as well as shutter tilting are evaluated.

The series of qualification testing are conducted at the MSA quadrant level. During the 2-D addressing test, the permanent magnet positioned on the backside of the shutter array (on the grid side) scans across the array. The scan opens shutters into the grid, column by column, to the position against back walls. The magnetic force can be adjusted by a programmed control of the distance between the shutter array and the magnet. Shutters are latched by applying voltages typically around 40V. A 2-D addressing is done by typically keeping $20 \mathrm{~V}$ at the selected shutters to hold them open, and turning off the bias to the rest of shutters to release them back to their closed position. The release process is synchronized with a sweep of the magnet so to gently send shutters back without impact to the light shields. Various 2$\mathrm{D}$ addressing patterns are programmed into the MSAs to test functional responses of shutters. A 2-D addressing pattern with programmed "ESA" letters in a MSA is shown in Figure 9. Shutters with transmitted light (bright) were latched open, while the rest (dark) were released and kept closed.

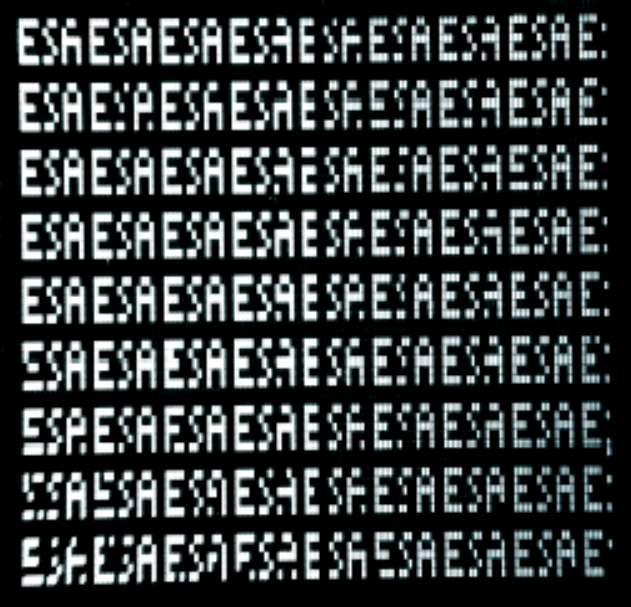

Figure 9. A 2-D addressing pattern from a flight-format $365 \times 171$ microshutter arrav showing "ESA" letters

Life cycling testing is conducted in a cryogenic chamber. MSAs tested at a cycling rate of $4 \mathrm{~Hz}$ passed 1 million cycles with minimal increase of failed open and failed closed shutters. Optical tests are performed to evaluate optical performance of MSAs, mainly the contrast. Shutter arrays fabricated with light shields are able to achieve a contrast of $>10,000$ that well exceeds the NIRSpec instrument requirement of 2000. Environmental tests consist of the radiation, acoustic and vibration tests. Radiation tests are conducted to simulate effects of ionizing particle radiation in space, while the acoustic and vibration tests are to simulate the environments at the JWST launching. MSAs we fabricated passed all the qualification tests.

\section{CURRENT STATUS AND FUTURE WORK}

Complete MSA quadrant assemblies have been successfully manufactured and fully functionally tested ${ }^{4}$. The assemblies have passed a series of critical reviews required by JWST project in satisfying all the design specifications.

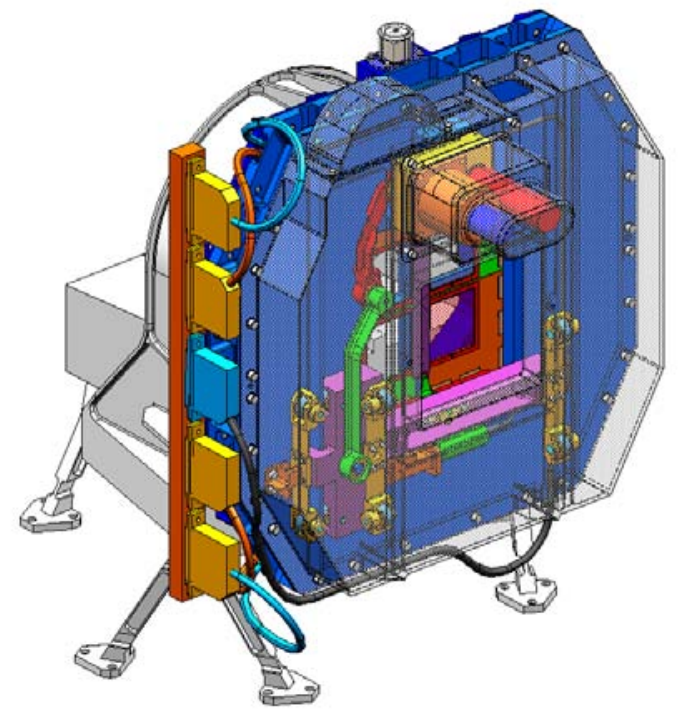

Figure 10. Final design of a microshutter array assembly with four MSA quadrants located in the center, a permanent magnet (pink) in up-down motion programmed to synchronize shutter open and close, step motors, and harnesses connected to the NIRSpec instrument

MSA teams are now making progress in final fabrication and testing the MSA system assembly (Figure 10). The delivery of flight-format MSA system is scheduled at the end of 2008 to be integrated to the focal plane of the NIRSpec detectors at ESA.

\section{ACKNOWLEDGEMENTS}

We express our appreciation to Drs. Reza Ghodssi, WenHsien Chuang and Mr. John Barry at UMPC, Dr. Shu-Fan Cheng at NRL, Mr. John Lehtonen and Ms. Katherine Mach at JHU/APL for their tremendous technical supports.

\section{REFERENCES}

${ }^{1}$ S. H. Moseley, et al, "Microshutter Arrays for the JWST Nearinfrared Spectrometer", Proc. SPIE Vol. 5487, pp. 645-652, Optical, Infrared, and Millimeter Space Telescopes; John C. Mather; Ed., SPIE conference "Astronomical Telescopes and Instrumentation 2004: Space Telescopes and Astronomy", Glasgow, Scotland 2004.

${ }^{2}$ M. Li, et al., invited paper "Microshutter Array Development for the James Webb Space Telescope", Smart Structures, Devices, and Systems II, Proc. SPIE, 5650, pp. 9-16, 2005.

${ }^{3}$ Christine A. Allen, et al, "Flip Chip Integration of a Large Format Array of MOEMS Devices for Space Infrared Astronomy", Proc. of the SMTA International Conference, Emerging Technologies Summit, 2006

${ }^{4} \mathrm{M}$. Li, et al, invited paper "Complex MEMS Device: Microshutter Array System for Space Applications", Micro (MEMS) and Nanotechnologies for Defense and Security, Proc. SPIE, 6556, 2007. 